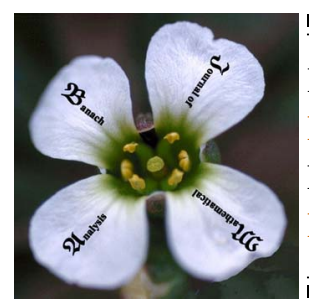

Banach J. Math. Anal. 10 (2016), no. 1, 71-95

http://dx.doi.org/10.1215/17358787-3319378

ISSN: $1735-8787$ (electronic)

http://projecteuclid.org/bjma

\title{
MULTIPLIERS AND HADAMARD PRODUCTS IN THE VECTOR-VALUED SETTING
}

\author{
ÓSCAR BLASCO and CARME ZARAGOZA-BERZOSA*
}

Communicated by R. Villena

\begin{abstract}
Let $E_{i}$ be Banach spaces, and let $X_{E_{i}}$ be Banach spaces continuously contained in the spaces of $E_{i}$-valued sequences $(\hat{x}(j))_{j} \in E_{i}^{\mathbb{N}}$, for $i=1,2,3$. Given a bounded bilinear map $B: E_{1} \times E_{2} \rightarrow E_{3}$, we define $\left(X_{E_{2}}, X_{E_{3}}\right)_{B}$, the space of $B$-multipliers between $X_{E_{2}}$ and $X_{E_{3}}$, to be the set of sequences $\left(\lambda_{j}\right)_{j} \in E_{1}^{\mathbb{N}}$ such that $\left(B\left(\lambda_{j}, \hat{x}(j)\right)\right)_{j} \in X_{E_{3}}$ for all $(\hat{x}(j))_{j} \in X_{E_{2}}$, and we define the Hadamard projective tensor product $X_{E_{1} \circledast_{B}} X_{E_{2}}$ as consisting of those elements in $E_{3}^{\mathbb{N}}$ that can be represented as $\sum_{n} \sum_{j} B\left(\hat{x}_{n}(j), \hat{y}_{n}(j)\right)$, where $\left(x_{n}\right)_{n} \in X_{E_{1}},\left(y_{n}\right)_{n} \in X_{E_{2}}$, and $\sum_{n}\left\|x_{n}\right\|_{X_{E_{1}}}\left\|y_{n}\right\|_{X_{E_{2}}}<\infty$.

We will analyze some properties of these two spaces, relate them, and compute the Hadamard tensor products and the spaces of vector-valued multipliers in several cases, getting applications in the particular case where $E=\mathcal{L}\left(E_{1}, E_{2}\right)$ and $B(T, x)=T(x)$.
\end{abstract}

\section{INTRODUCTION AND PRELIMINARIES}

One of the classic problems in Fourier analysis is the description of the space of coefficient multipliers between function spaces. Several papers have shown mathematicians' interest in determining this space in particular cases (see the recent monograph [18]; see also [20] for the historical situation for Hardy spaces and [16] and [17] for several techniques and results regarding mixed norm; we refer the

Copyright 2016 by the Tusi Mathematical Research Group.

Received Dec. 11, 2014; Accepted Apr. 14, 2015.

${ }^{*}$ Corresponding author.

2010 Mathematics Subject Classification. Primary 46B28; Secondary 46E40.

Keywords. vector-valued multipliers, Hadamard product, bilinear map. 


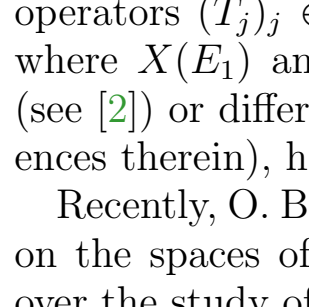

Banach J. Math. Anal. 10 (2016), no. 1, 71-95

http://dx.doi.org/10.1215/17358787-3319378

ISSN: $1735-8787$ (electronic)

http://projecteuclid.org/bjma

\title{
MULTIPLIERS AND HADAMARD PRODUCTS IN THE VECTOR-VALUED SETTING
}

\author{
ÓSCAR BLASCO and CARME ZARAGOZA-BERZOSA*
}

Communicated by R. Villena

\begin{abstract}
Let $E_{i}$ be Banach spaces, and let $X_{E_{i}}$ be Banach spaces continuously contained in the spaces of $E_{i}$-valued sequences $(\hat{x}(j))_{j} \in E_{i}^{\mathbb{N}}$, for $i=1,2,3$. Given a bounded bilinear map $B: E_{1} \times E_{2} \rightarrow E_{3}$, we define $\left(X_{E_{2}}, X_{E_{3}}\right)_{B}$, the space of $B$-multipliers between $X_{E_{2}}$ and $X_{E_{3}}$, to be the set of sequences $\left(\lambda_{j}\right)_{j} \in E_{1}^{\mathbb{N}}$ such that $\left(B\left(\lambda_{j}, \hat{x}(j)\right)\right)_{j} \in X_{E_{3}}$ for all $(\hat{x}(j))_{j} \in X_{E_{2}}$, and we define the Hadamard projective tensor product $X_{E_{1} \circledast_{B}} X_{E_{2}}$ as consisting of those elements in $E_{3}^{\mathbb{N}}$ that can be represented as $\sum_{n} \sum_{j} B\left(\hat{x}_{n}(j), \hat{y}_{n}(j)\right)$, where $\left(x_{n}\right)_{n} \in X_{E_{1}},\left(y_{n}\right)_{n} \in X_{E_{2}}$, and $\sum_{n}\left\|x_{n}\right\|_{X_{E_{1}}}\left\|y_{n}\right\|_{X_{E_{2}}}<\infty$.

We will analyze some properties of these two spaces, relate them, and compute the Hadamard tensor products and the spaces of vector-valued multipliers in several cases, getting applications in the particular case where $E=\mathcal{L}\left(E_{1}, E_{2}\right)$ and $B(T, x)=T(x)$.
\end{abstract}

\section{INTRODUCTION AND PRELIMINARIES}

One of the classic problems in Fourier analysis is the description of the space of coefficient multipliers between function spaces. Several papers have shown mathematicians' interest in determining this space in particular cases (see the recent monograph [18]; see also [20] for the historical situation for Hardy spaces and [16] and [17] for several techniques and results regarding mixed norm; we refer the

Copyright 2016 by the Tusi Mathematical Research Group.

Received Dec. 11, 2014; Accepted Apr. 14, 2015.

${ }^{*}$ Corresponding author.

2010 Mathematics Subject Classification. Primary 46B28; Secondary 46E40.

Keywords. vector-valued multipliers, Hadamard product, bilinear map. 


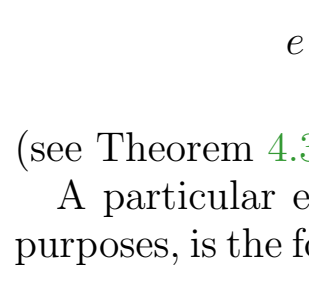

Banach J. Math. Anal. 10 (2016), no. 1, 71-95

http://dx.doi.org/10.1215/17358787-3319378

ISSN: $1735-8787$ (electronic)

http://projecteuclid.org/bjma

\title{
MULTIPLIERS AND HADAMARD PRODUCTS IN THE VECTOR-VALUED SETTING
}

\author{
ÓSCAR BLASCO and CARME ZARAGOZA-BERZOSA*
}

Communicated by R. Villena

\begin{abstract}
Let $E_{i}$ be Banach spaces, and let $X_{E_{i}}$ be Banach spaces continuously contained in the spaces of $E_{i}$-valued sequences $(\hat{x}(j))_{j} \in E_{i}^{\mathbb{N}}$, for $i=1,2,3$. Given a bounded bilinear map $B: E_{1} \times E_{2} \rightarrow E_{3}$, we define $\left(X_{E_{2}}, X_{E_{3}}\right)_{B}$, the space of $B$-multipliers between $X_{E_{2}}$ and $X_{E_{3}}$, to be the set of sequences $\left(\lambda_{j}\right)_{j} \in E_{1}^{\mathbb{N}}$ such that $\left(B\left(\lambda_{j}, \hat{x}(j)\right)\right)_{j} \in X_{E_{3}}$ for all $(\hat{x}(j))_{j} \in X_{E_{2}}$, and we define the Hadamard projective tensor product $X_{E_{1} \circledast_{B}} X_{E_{2}}$ as consisting of those elements in $E_{3}^{\mathbb{N}}$ that can be represented as $\sum_{n} \sum_{j} B\left(\hat{x}_{n}(j), \hat{y}_{n}(j)\right)$, where $\left(x_{n}\right)_{n} \in X_{E_{1}},\left(y_{n}\right)_{n} \in X_{E_{2}}$, and $\sum_{n}\left\|x_{n}\right\|_{X_{E_{1}}}\left\|y_{n}\right\|_{X_{E_{2}}}<\infty$.

We will analyze some properties of these two spaces, relate them, and compute the Hadamard tensor products and the spaces of vector-valued multipliers in several cases, getting applications in the particular case where $E=\mathcal{L}\left(E_{1}, E_{2}\right)$ and $B(T, x)=T(x)$.
\end{abstract}

\section{INTRODUCTION AND PRELIMINARIES}

One of the classic problems in Fourier analysis is the description of the space of coefficient multipliers between function spaces. Several papers have shown mathematicians' interest in determining this space in particular cases (see the recent monograph [18]; see also [20] for the historical situation for Hardy spaces and [16] and [17] for several techniques and results regarding mixed norm; we refer the

Copyright 2016 by the Tusi Mathematical Research Group.

Received Dec. 11, 2014; Accepted Apr. 14, 2015.

${ }^{*}$ Corresponding author.

2010 Mathematics Subject Classification. Primary 46B28; Secondary 46E40.

Keywords. vector-valued multipliers, Hadamard product, bilinear map. 


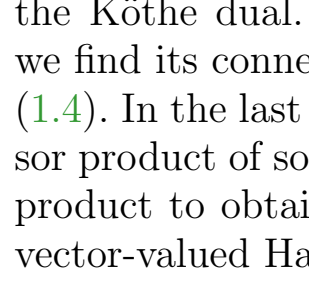

Banach J. Math. Anal. 10 (2016), no. 1, 71-95

http://dx.doi.org/10.1215/17358787-3319378

ISSN: $1735-8787$ (electronic)

http://projecteuclid.org/bjma

\title{
MULTIPLIERS AND HADAMARD PRODUCTS IN THE VECTOR-VALUED SETTING
}

\author{
ÓSCAR BLASCO and CARME ZARAGOZA-BERZOSA*
}

Communicated by R. Villena

\begin{abstract}
Let $E_{i}$ be Banach spaces, and let $X_{E_{i}}$ be Banach spaces continuously contained in the spaces of $E_{i}$-valued sequences $(\hat{x}(j))_{j} \in E_{i}^{\mathbb{N}}$, for $i=1,2,3$. Given a bounded bilinear map $B: E_{1} \times E_{2} \rightarrow E_{3}$, we define $\left(X_{E_{2}}, X_{E_{3}}\right)_{B}$, the space of $B$-multipliers between $X_{E_{2}}$ and $X_{E_{3}}$, to be the set of sequences $\left(\lambda_{j}\right)_{j} \in E_{1}^{\mathbb{N}}$ such that $\left(B\left(\lambda_{j}, \hat{x}(j)\right)\right)_{j} \in X_{E_{3}}$ for all $(\hat{x}(j))_{j} \in X_{E_{2}}$, and we define the Hadamard projective tensor product $X_{E_{1} \circledast_{B}} X_{E_{2}}$ as consisting of those elements in $E_{3}^{\mathbb{N}}$ that can be represented as $\sum_{n} \sum_{j} B\left(\hat{x}_{n}(j), \hat{y}_{n}(j)\right)$, where $\left(x_{n}\right)_{n} \in X_{E_{1}},\left(y_{n}\right)_{n} \in X_{E_{2}}$, and $\sum_{n}\left\|x_{n}\right\|_{X_{E_{1}}}\left\|y_{n}\right\|_{X_{E_{2}}}<\infty$.

We will analyze some properties of these two spaces, relate them, and compute the Hadamard tensor products and the spaces of vector-valued multipliers in several cases, getting applications in the particular case where $E=\mathcal{L}\left(E_{1}, E_{2}\right)$ and $B(T, x)=T(x)$.
\end{abstract}

\section{INTRODUCTION AND PRELIMINARIES}

One of the classic problems in Fourier analysis is the description of the space of coefficient multipliers between function spaces. Several papers have shown mathematicians' interest in determining this space in particular cases (see the recent monograph [18]; see also [20] for the historical situation for Hardy spaces and [16] and [17] for several techniques and results regarding mixed norm; we refer the

Copyright 2016 by the Tusi Mathematical Research Group.

Received Dec. 11, 2014; Accepted Apr. 14, 2015.

${ }^{*}$ Corresponding author.

2010 Mathematics Subject Classification. Primary 46B28; Secondary 46E40.

Keywords. vector-valued multipliers, Hadamard product, bilinear map. 


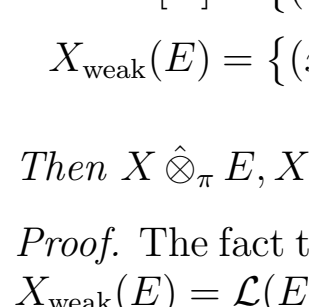

Banach J. Math. Anal. 10 (2016), no. 1, 71-95

http://dx.doi.org/10.1215/17358787-3319378

ISSN: $1735-8787$ (electronic)

http://projecteuclid.org/bjma

\title{
MULTIPLIERS AND HADAMARD PRODUCTS IN THE VECTOR-VALUED SETTING
}

\author{
ÓSCAR BLASCO and CARME ZARAGOZA-BERZOSA*
}

Communicated by R. Villena

\begin{abstract}
Let $E_{i}$ be Banach spaces, and let $X_{E_{i}}$ be Banach spaces continuously contained in the spaces of $E_{i}$-valued sequences $(\hat{x}(j))_{j} \in E_{i}^{\mathbb{N}}$, for $i=1,2,3$. Given a bounded bilinear map $B: E_{1} \times E_{2} \rightarrow E_{3}$, we define $\left(X_{E_{2}}, X_{E_{3}}\right)_{B}$, the space of $B$-multipliers between $X_{E_{2}}$ and $X_{E_{3}}$, to be the set of sequences $\left(\lambda_{j}\right)_{j} \in E_{1}^{\mathbb{N}}$ such that $\left(B\left(\lambda_{j}, \hat{x}(j)\right)\right)_{j} \in X_{E_{3}}$ for all $(\hat{x}(j))_{j} \in X_{E_{2}}$, and we define the Hadamard projective tensor product $X_{E_{1} \circledast_{B}} X_{E_{2}}$ as consisting of those elements in $E_{3}^{\mathbb{N}}$ that can be represented as $\sum_{n} \sum_{j} B\left(\hat{x}_{n}(j), \hat{y}_{n}(j)\right)$, where $\left(x_{n}\right)_{n} \in X_{E_{1}},\left(y_{n}\right)_{n} \in X_{E_{2}}$, and $\sum_{n}\left\|x_{n}\right\|_{X_{E_{1}}}\left\|y_{n}\right\|_{X_{E_{2}}}<\infty$.

We will analyze some properties of these two spaces, relate them, and compute the Hadamard tensor products and the spaces of vector-valued multipliers in several cases, getting applications in the particular case where $E=\mathcal{L}\left(E_{1}, E_{2}\right)$ and $B(T, x)=T(x)$.
\end{abstract}

\section{INTRODUCTION AND PRELIMINARIES}

One of the classic problems in Fourier analysis is the description of the space of coefficient multipliers between function spaces. Several papers have shown mathematicians' interest in determining this space in particular cases (see the recent monograph [18]; see also [20] for the historical situation for Hardy spaces and [16] and [17] for several techniques and results regarding mixed norm; we refer the

Copyright 2016 by the Tusi Mathematical Research Group.

Received Dec. 11, 2014; Accepted Apr. 14, 2015.

${ }^{*}$ Corresponding author.

2010 Mathematics Subject Classification. Primary 46B28; Secondary 46E40.

Keywords. vector-valued multipliers, Hadamard product, bilinear map. 


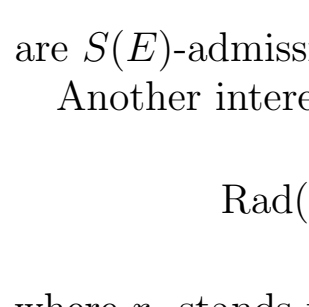

Banach J. Math. Anal. 10 (2016), no. 1, 71-95

http://dx.doi.org/10.1215/17358787-3319378

ISSN: $1735-8787$ (electronic)

http://projecteuclid.org/bjma

\title{
MULTIPLIERS AND HADAMARD PRODUCTS IN THE VECTOR-VALUED SETTING
}

\author{
ÓSCAR BLASCO and CARME ZARAGOZA-BERZOSA*
}

Communicated by R. Villena

\begin{abstract}
Let $E_{i}$ be Banach spaces, and let $X_{E_{i}}$ be Banach spaces continuously contained in the spaces of $E_{i}$-valued sequences $(\hat{x}(j))_{j} \in E_{i}^{\mathbb{N}}$, for $i=1,2,3$. Given a bounded bilinear map $B: E_{1} \times E_{2} \rightarrow E_{3}$, we define $\left(X_{E_{2}}, X_{E_{3}}\right)_{B}$, the space of $B$-multipliers between $X_{E_{2}}$ and $X_{E_{3}}$, to be the set of sequences $\left(\lambda_{j}\right)_{j} \in E_{1}^{\mathbb{N}}$ such that $\left(B\left(\lambda_{j}, \hat{x}(j)\right)\right)_{j} \in X_{E_{3}}$ for all $(\hat{x}(j))_{j} \in X_{E_{2}}$, and we define the Hadamard projective tensor product $X_{E_{1} \circledast_{B}} X_{E_{2}}$ as consisting of those elements in $E_{3}^{\mathbb{N}}$ that can be represented as $\sum_{n} \sum_{j} B\left(\hat{x}_{n}(j), \hat{y}_{n}(j)\right)$, where $\left(x_{n}\right)_{n} \in X_{E_{1}},\left(y_{n}\right)_{n} \in X_{E_{2}}$, and $\sum_{n}\left\|x_{n}\right\|_{X_{E_{1}}}\left\|y_{n}\right\|_{X_{E_{2}}}<\infty$.

We will analyze some properties of these two spaces, relate them, and compute the Hadamard tensor products and the spaces of vector-valued multipliers in several cases, getting applications in the particular case where $E=\mathcal{L}\left(E_{1}, E_{2}\right)$ and $B(T, x)=T(x)$.
\end{abstract}

\section{INTRODUCTION AND PRELIMINARIES}

One of the classic problems in Fourier analysis is the description of the space of coefficient multipliers between function spaces. Several papers have shown mathematicians' interest in determining this space in particular cases (see the recent monograph [18]; see also [20] for the historical situation for Hardy spaces and [16] and [17] for several techniques and results regarding mixed norm; we refer the

Copyright 2016 by the Tusi Mathematical Research Group.

Received Dec. 11, 2014; Accepted Apr. 14, 2015.

${ }^{*}$ Corresponding author.

2010 Mathematics Subject Classification. Primary 46B28; Secondary 46E40.

Keywords. vector-valued multipliers, Hadamard product, bilinear map. 


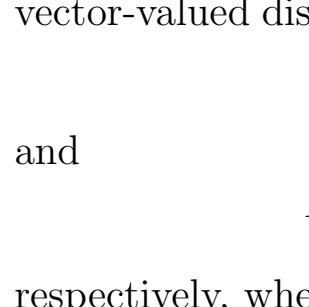

Banach J. Math. Anal. 10 (2016), no. 1, 71-95

http://dx.doi.org/10.1215/17358787-3319378

ISSN: $1735-8787$ (electronic)

http://projecteuclid.org/bjma

\title{
MULTIPLIERS AND HADAMARD PRODUCTS IN THE VECTOR-VALUED SETTING
}

\author{
ÓSCAR BLASCO and CARME ZARAGOZA-BERZOSA*
}

Communicated by R. Villena

\begin{abstract}
Let $E_{i}$ be Banach spaces, and let $X_{E_{i}}$ be Banach spaces continuously contained in the spaces of $E_{i}$-valued sequences $(\hat{x}(j))_{j} \in E_{i}^{\mathbb{N}}$, for $i=1,2,3$. Given a bounded bilinear map $B: E_{1} \times E_{2} \rightarrow E_{3}$, we define $\left(X_{E_{2}}, X_{E_{3}}\right)_{B}$, the space of $B$-multipliers between $X_{E_{2}}$ and $X_{E_{3}}$, to be the set of sequences $\left(\lambda_{j}\right)_{j} \in E_{1}^{\mathbb{N}}$ such that $\left(B\left(\lambda_{j}, \hat{x}(j)\right)\right)_{j} \in X_{E_{3}}$ for all $(\hat{x}(j))_{j} \in X_{E_{2}}$, and we define the Hadamard projective tensor product $X_{E_{1} \circledast_{B}} X_{E_{2}}$ as consisting of those elements in $E_{3}^{\mathbb{N}}$ that can be represented as $\sum_{n} \sum_{j} B\left(\hat{x}_{n}(j), \hat{y}_{n}(j)\right)$, where $\left(x_{n}\right)_{n} \in X_{E_{1}},\left(y_{n}\right)_{n} \in X_{E_{2}}$, and $\sum_{n}\left\|x_{n}\right\|_{X_{E_{1}}}\left\|y_{n}\right\|_{X_{E_{2}}}<\infty$.

We will analyze some properties of these two spaces, relate them, and compute the Hadamard tensor products and the spaces of vector-valued multipliers in several cases, getting applications in the particular case where $E=\mathcal{L}\left(E_{1}, E_{2}\right)$ and $B(T, x)=T(x)$.
\end{abstract}

\section{INTRODUCTION AND PRELIMINARIES}

One of the classic problems in Fourier analysis is the description of the space of coefficient multipliers between function spaces. Several papers have shown mathematicians' interest in determining this space in particular cases (see the recent monograph [18]; see also [20] for the historical situation for Hardy spaces and [16] and [17] for several techniques and results regarding mixed norm; we refer the

Copyright 2016 by the Tusi Mathematical Research Group.

Received Dec. 11, 2014; Accepted Apr. 14, 2015.

${ }^{*}$ Corresponding author.

2010 Mathematics Subject Classification. Primary 46B28; Secondary 46E40.

Keywords. vector-valued multipliers, Hadamard product, bilinear map. 


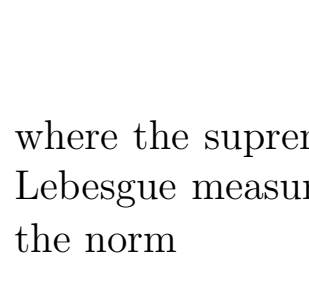

Banach J. Math. Anal. 10 (2016), no. 1, 71-95

http://dx.doi.org/10.1215/17358787-3319378

ISSN: $1735-8787$ (electronic)

http://projecteuclid.org/bjma

\title{
MULTIPLIERS AND HADAMARD PRODUCTS IN THE VECTOR-VALUED SETTING
}

\author{
ÓSCAR BLASCO and CARME ZARAGOZA-BERZOSA*
}

Communicated by R. Villena

\begin{abstract}
Let $E_{i}$ be Banach spaces, and let $X_{E_{i}}$ be Banach spaces continuously contained in the spaces of $E_{i}$-valued sequences $(\hat{x}(j))_{j} \in E_{i}^{\mathbb{N}}$, for $i=1,2,3$. Given a bounded bilinear map $B: E_{1} \times E_{2} \rightarrow E_{3}$, we define $\left(X_{E_{2}}, X_{E_{3}}\right)_{B}$, the space of $B$-multipliers between $X_{E_{2}}$ and $X_{E_{3}}$, to be the set of sequences $\left(\lambda_{j}\right)_{j} \in E_{1}^{\mathbb{N}}$ such that $\left(B\left(\lambda_{j}, \hat{x}(j)\right)\right)_{j} \in X_{E_{3}}$ for all $(\hat{x}(j))_{j} \in X_{E_{2}}$, and we define the Hadamard projective tensor product $X_{E_{1} \circledast_{B}} X_{E_{2}}$ as consisting of those elements in $E_{3}^{\mathbb{N}}$ that can be represented as $\sum_{n} \sum_{j} B\left(\hat{x}_{n}(j), \hat{y}_{n}(j)\right)$, where $\left(x_{n}\right)_{n} \in X_{E_{1}},\left(y_{n}\right)_{n} \in X_{E_{2}}$, and $\sum_{n}\left\|x_{n}\right\|_{X_{E_{1}}}\left\|y_{n}\right\|_{X_{E_{2}}}<\infty$.

We will analyze some properties of these two spaces, relate them, and compute the Hadamard tensor products and the spaces of vector-valued multipliers in several cases, getting applications in the particular case where $E=\mathcal{L}\left(E_{1}, E_{2}\right)$ and $B(T, x)=T(x)$.
\end{abstract}

\section{INTRODUCTION AND PRELIMINARIES}

One of the classic problems in Fourier analysis is the description of the space of coefficient multipliers between function spaces. Several papers have shown mathematicians' interest in determining this space in particular cases (see the recent monograph [18]; see also [20] for the historical situation for Hardy spaces and [16] and [17] for several techniques and results regarding mixed norm; we refer the

Copyright 2016 by the Tusi Mathematical Research Group.

Received Dec. 11, 2014; Accepted Apr. 14, 2015.

${ }^{*}$ Corresponding author.

2010 Mathematics Subject Classification. Primary 46B28; Secondary 46E40.

Keywords. vector-valued multipliers, Hadamard product, bilinear map. 


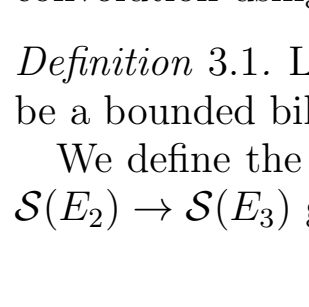

Banach J. Math. Anal. 10 (2016), no. 1, 71-95

http://dx.doi.org/10.1215/17358787-3319378

ISSN: $1735-8787$ (electronic)

http://projecteuclid.org/bjma

\title{
MULTIPLIERS AND HADAMARD PRODUCTS IN THE VECTOR-VALUED SETTING
}

\author{
ÓSCAR BLASCO and CARME ZARAGOZA-BERZOSA*
}

Communicated by R. Villena

\begin{abstract}
Let $E_{i}$ be Banach spaces, and let $X_{E_{i}}$ be Banach spaces continuously contained in the spaces of $E_{i}$-valued sequences $(\hat{x}(j))_{j} \in E_{i}^{\mathbb{N}}$, for $i=1,2,3$. Given a bounded bilinear map $B: E_{1} \times E_{2} \rightarrow E_{3}$, we define $\left(X_{E_{2}}, X_{E_{3}}\right)_{B}$, the space of $B$-multipliers between $X_{E_{2}}$ and $X_{E_{3}}$, to be the set of sequences $\left(\lambda_{j}\right)_{j} \in E_{1}^{\mathbb{N}}$ such that $\left(B\left(\lambda_{j}, \hat{x}(j)\right)\right)_{j} \in X_{E_{3}}$ for all $(\hat{x}(j))_{j} \in X_{E_{2}}$, and we define the Hadamard projective tensor product $X_{E_{1} \circledast_{B}} X_{E_{2}}$ as consisting of those elements in $E_{3}^{\mathbb{N}}$ that can be represented as $\sum_{n} \sum_{j} B\left(\hat{x}_{n}(j), \hat{y}_{n}(j)\right)$, where $\left(x_{n}\right)_{n} \in X_{E_{1}},\left(y_{n}\right)_{n} \in X_{E_{2}}$, and $\sum_{n}\left\|x_{n}\right\|_{X_{E_{1}}}\left\|y_{n}\right\|_{X_{E_{2}}}<\infty$.

We will analyze some properties of these two spaces, relate them, and compute the Hadamard tensor products and the spaces of vector-valued multipliers in several cases, getting applications in the particular case where $E=\mathcal{L}\left(E_{1}, E_{2}\right)$ and $B(T, x)=T(x)$.
\end{abstract}

\section{INTRODUCTION AND PRELIMINARIES}

One of the classic problems in Fourier analysis is the description of the space of coefficient multipliers between function spaces. Several papers have shown mathematicians' interest in determining this space in particular cases (see the recent monograph [18]; see also [20] for the historical situation for Hardy spaces and [16] and [17] for several techniques and results regarding mixed norm; we refer the

Copyright 2016 by the Tusi Mathematical Research Group.

Received Dec. 11, 2014; Accepted Apr. 14, 2015.

${ }^{*}$ Corresponding author.

2010 Mathematics Subject Classification. Primary 46B28; Secondary 46E40.

Keywords. vector-valued multipliers, Hadamard product, bilinear map. 


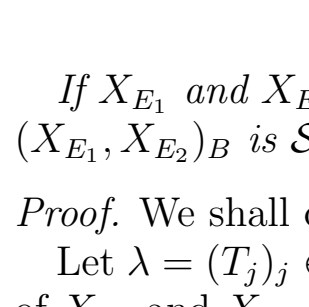

Banach J. Math. Anal. 10 (2016), no. 1, 71-95

http://dx.doi.org/10.1215/17358787-3319378

ISSN: $1735-8787$ (electronic)

http://projecteuclid.org/bjma

\title{
MULTIPLIERS AND HADAMARD PRODUCTS IN THE VECTOR-VALUED SETTING
}

\author{
ÓSCAR BLASCO and CARME ZARAGOZA-BERZOSA*
}

Communicated by R. Villena

\begin{abstract}
Let $E_{i}$ be Banach spaces, and let $X_{E_{i}}$ be Banach spaces continuously contained in the spaces of $E_{i}$-valued sequences $(\hat{x}(j))_{j} \in E_{i}^{\mathbb{N}}$, for $i=1,2,3$. Given a bounded bilinear map $B: E_{1} \times E_{2} \rightarrow E_{3}$, we define $\left(X_{E_{2}}, X_{E_{3}}\right)_{B}$, the space of $B$-multipliers between $X_{E_{2}}$ and $X_{E_{3}}$, to be the set of sequences $\left(\lambda_{j}\right)_{j} \in E_{1}^{\mathbb{N}}$ such that $\left(B\left(\lambda_{j}, \hat{x}(j)\right)\right)_{j} \in X_{E_{3}}$ for all $(\hat{x}(j))_{j} \in X_{E_{2}}$, and we define the Hadamard projective tensor product $X_{E_{1} \circledast_{B}} X_{E_{2}}$ as consisting of those elements in $E_{3}^{\mathbb{N}}$ that can be represented as $\sum_{n} \sum_{j} B\left(\hat{x}_{n}(j), \hat{y}_{n}(j)\right)$, where $\left(x_{n}\right)_{n} \in X_{E_{1}},\left(y_{n}\right)_{n} \in X_{E_{2}}$, and $\sum_{n}\left\|x_{n}\right\|_{X_{E_{1}}}\left\|y_{n}\right\|_{X_{E_{2}}}<\infty$.

We will analyze some properties of these two spaces, relate them, and compute the Hadamard tensor products and the spaces of vector-valued multipliers in several cases, getting applications in the particular case where $E=\mathcal{L}\left(E_{1}, E_{2}\right)$ and $B(T, x)=T(x)$.
\end{abstract}

\section{INTRODUCTION AND PRELIMINARIES}

One of the classic problems in Fourier analysis is the description of the space of coefficient multipliers between function spaces. Several papers have shown mathematicians' interest in determining this space in particular cases (see the recent monograph [18]; see also [20] for the historical situation for Hardy spaces and [16] and [17] for several techniques and results regarding mixed norm; we refer the

Copyright 2016 by the Tusi Mathematical Research Group.

Received Dec. 11, 2014; Accepted Apr. 14, 2015.

${ }^{*}$ Corresponding author.

2010 Mathematics Subject Classification. Primary 46B28; Secondary 46E40.

Keywords. vector-valued multipliers, Hadamard product, bilinear map. 


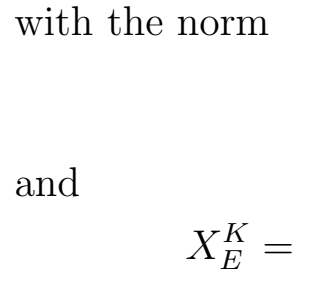

Banach J. Math. Anal. 10 (2016), no. 1, 71-95

http://dx.doi.org/10.1215/17358787-3319378

ISSN: $1735-8787$ (electronic)

http://projecteuclid.org/bjma

\title{
MULTIPLIERS AND HADAMARD PRODUCTS IN THE VECTOR-VALUED SETTING
}

\author{
ÓSCAR BLASCO and CARME ZARAGOZA-BERZOSA*
}

Communicated by R. Villena

\begin{abstract}
Let $E_{i}$ be Banach spaces, and let $X_{E_{i}}$ be Banach spaces continuously contained in the spaces of $E_{i}$-valued sequences $(\hat{x}(j))_{j} \in E_{i}^{\mathbb{N}}$, for $i=1,2,3$. Given a bounded bilinear map $B: E_{1} \times E_{2} \rightarrow E_{3}$, we define $\left(X_{E_{2}}, X_{E_{3}}\right)_{B}$, the space of $B$-multipliers between $X_{E_{2}}$ and $X_{E_{3}}$, to be the set of sequences $\left(\lambda_{j}\right)_{j} \in E_{1}^{\mathbb{N}}$ such that $\left(B\left(\lambda_{j}, \hat{x}(j)\right)\right)_{j} \in X_{E_{3}}$ for all $(\hat{x}(j))_{j} \in X_{E_{2}}$, and we define the Hadamard projective tensor product $X_{E_{1} \circledast_{B}} X_{E_{2}}$ as consisting of those elements in $E_{3}^{\mathbb{N}}$ that can be represented as $\sum_{n} \sum_{j} B\left(\hat{x}_{n}(j), \hat{y}_{n}(j)\right)$, where $\left(x_{n}\right)_{n} \in X_{E_{1}},\left(y_{n}\right)_{n} \in X_{E_{2}}$, and $\sum_{n}\left\|x_{n}\right\|_{X_{E_{1}}}\left\|y_{n}\right\|_{X_{E_{2}}}<\infty$.

We will analyze some properties of these two spaces, relate them, and compute the Hadamard tensor products and the spaces of vector-valued multipliers in several cases, getting applications in the particular case where $E=\mathcal{L}\left(E_{1}, E_{2}\right)$ and $B(T, x)=T(x)$.
\end{abstract}

\section{INTRODUCTION AND PRELIMINARIES}

One of the classic problems in Fourier analysis is the description of the space of coefficient multipliers between function spaces. Several papers have shown mathematicians' interest in determining this space in particular cases (see the recent monograph [18]; see also [20] for the historical situation for Hardy spaces and [16] and [17] for several techniques and results regarding mixed norm; we refer the

Copyright 2016 by the Tusi Mathematical Research Group.

Received Dec. 11, 2014; Accepted Apr. 14, 2015.

${ }^{*}$ Corresponding author.

2010 Mathematics Subject Classification. Primary 46B28; Secondary 46E40.

Keywords. vector-valued multipliers, Hadamard product, bilinear map. 


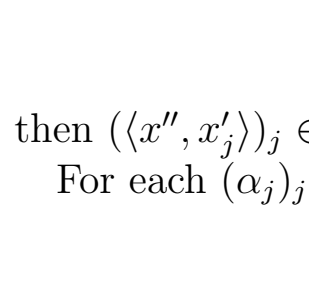

Banach J. Math. Anal. 10 (2016), no. 1, 71-95

http://dx.doi.org/10.1215/17358787-3319378

ISSN: $1735-8787$ (electronic)

http://projecteuclid.org/bjma

\title{
MULTIPLIERS AND HADAMARD PRODUCTS IN THE VECTOR-VALUED SETTING
}

\author{
ÓSCAR BLASCO and CARME ZARAGOZA-BERZOSA*
}

Communicated by R. Villena

\begin{abstract}
Let $E_{i}$ be Banach spaces, and let $X_{E_{i}}$ be Banach spaces continuously contained in the spaces of $E_{i}$-valued sequences $(\hat{x}(j))_{j} \in E_{i}^{\mathbb{N}}$, for $i=1,2,3$. Given a bounded bilinear map $B: E_{1} \times E_{2} \rightarrow E_{3}$, we define $\left(X_{E_{2}}, X_{E_{3}}\right)_{B}$, the space of $B$-multipliers between $X_{E_{2}}$ and $X_{E_{3}}$, to be the set of sequences $\left(\lambda_{j}\right)_{j} \in E_{1}^{\mathbb{N}}$ such that $\left(B\left(\lambda_{j}, \hat{x}(j)\right)\right)_{j} \in X_{E_{3}}$ for all $(\hat{x}(j))_{j} \in X_{E_{2}}$, and we define the Hadamard projective tensor product $X_{E_{1} \circledast_{B}} X_{E_{2}}$ as consisting of those elements in $E_{3}^{\mathbb{N}}$ that can be represented as $\sum_{n} \sum_{j} B\left(\hat{x}_{n}(j), \hat{y}_{n}(j)\right)$, where $\left(x_{n}\right)_{n} \in X_{E_{1}},\left(y_{n}\right)_{n} \in X_{E_{2}}$, and $\sum_{n}\left\|x_{n}\right\|_{X_{E_{1}}}\left\|y_{n}\right\|_{X_{E_{2}}}<\infty$.

We will analyze some properties of these two spaces, relate them, and compute the Hadamard tensor products and the spaces of vector-valued multipliers in several cases, getting applications in the particular case where $E=\mathcal{L}\left(E_{1}, E_{2}\right)$ and $B(T, x)=T(x)$.
\end{abstract}

\section{INTRODUCTION AND PRELIMINARIES}

One of the classic problems in Fourier analysis is the description of the space of coefficient multipliers between function spaces. Several papers have shown mathematicians' interest in determining this space in particular cases (see the recent monograph [18]; see also [20] for the historical situation for Hardy spaces and [16] and [17] for several techniques and results regarding mixed norm; we refer the

Copyright 2016 by the Tusi Mathematical Research Group.

Received Dec. 11, 2014; Accepted Apr. 14, 2015.

${ }^{*}$ Corresponding author.

2010 Mathematics Subject Classification. Primary 46B28; Secondary 46E40.

Keywords. vector-valued multipliers, Hadamard product, bilinear map. 


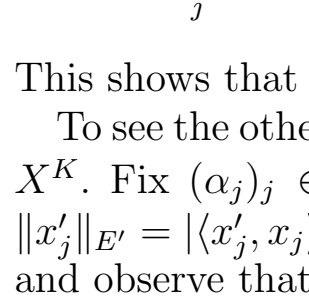

Banach J. Math. Anal. 10 (2016), no. 1, 71-95

http://dx.doi.org/10.1215/17358787-3319378

ISSN: $1735-8787$ (electronic)

http://projecteuclid.org/bjma

\title{
MULTIPLIERS AND HADAMARD PRODUCTS IN THE VECTOR-VALUED SETTING
}

\author{
ÓSCAR BLASCO and CARME ZARAGOZA-BERZOSA*
}

Communicated by R. Villena

\begin{abstract}
Let $E_{i}$ be Banach spaces, and let $X_{E_{i}}$ be Banach spaces continuously contained in the spaces of $E_{i}$-valued sequences $(\hat{x}(j))_{j} \in E_{i}^{\mathbb{N}}$, for $i=1,2,3$. Given a bounded bilinear map $B: E_{1} \times E_{2} \rightarrow E_{3}$, we define $\left(X_{E_{2}}, X_{E_{3}}\right)_{B}$, the space of $B$-multipliers between $X_{E_{2}}$ and $X_{E_{3}}$, to be the set of sequences $\left(\lambda_{j}\right)_{j} \in E_{1}^{\mathbb{N}}$ such that $\left(B\left(\lambda_{j}, \hat{x}(j)\right)\right)_{j} \in X_{E_{3}}$ for all $(\hat{x}(j))_{j} \in X_{E_{2}}$, and we define the Hadamard projective tensor product $X_{E_{1} \circledast_{B}} X_{E_{2}}$ as consisting of those elements in $E_{3}^{\mathbb{N}}$ that can be represented as $\sum_{n} \sum_{j} B\left(\hat{x}_{n}(j), \hat{y}_{n}(j)\right)$, where $\left(x_{n}\right)_{n} \in X_{E_{1}},\left(y_{n}\right)_{n} \in X_{E_{2}}$, and $\sum_{n}\left\|x_{n}\right\|_{X_{E_{1}}}\left\|y_{n}\right\|_{X_{E_{2}}}<\infty$.

We will analyze some properties of these two spaces, relate them, and compute the Hadamard tensor products and the spaces of vector-valued multipliers in several cases, getting applications in the particular case where $E=\mathcal{L}\left(E_{1}, E_{2}\right)$ and $B(T, x)=T(x)$.
\end{abstract}

\section{INTRODUCTION AND PRELIMINARIES}

One of the classic problems in Fourier analysis is the description of the space of coefficient multipliers between function spaces. Several papers have shown mathematicians' interest in determining this space in particular cases (see the recent monograph [18]; see also [20] for the historical situation for Hardy spaces and [16] and [17] for several techniques and results regarding mixed norm; we refer the

Copyright 2016 by the Tusi Mathematical Research Group.

Received Dec. 11, 2014; Accepted Apr. 14, 2015.

${ }^{*}$ Corresponding author.

2010 Mathematics Subject Classification. Primary 46B28; Secondary 46E40.

Keywords. vector-valued multipliers, Hadamard product, bilinear map. 


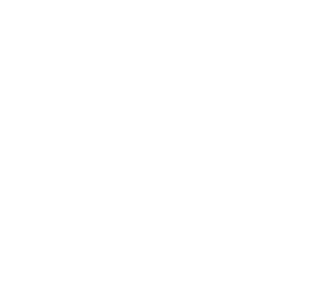

Banach J. Math. Anal. 10 (2016), no. 1, 71-95

http://dx.doi.org/10.1215/17358787-3319378

ISSN: $1735-8787$ (electronic)

http://projecteuclid.org/bjma

\title{
MULTIPLIERS AND HADAMARD PRODUCTS IN THE VECTOR-VALUED SETTING
}

\author{
ÓSCAR BLASCO and CARME ZARAGOZA-BERZOSA*
}

Communicated by R. Villena

\begin{abstract}
Let $E_{i}$ be Banach spaces, and let $X_{E_{i}}$ be Banach spaces continuously contained in the spaces of $E_{i}$-valued sequences $(\hat{x}(j))_{j} \in E_{i}^{\mathbb{N}}$, for $i=1,2,3$. Given a bounded bilinear map $B: E_{1} \times E_{2} \rightarrow E_{3}$, we define $\left(X_{E_{2}}, X_{E_{3}}\right)_{B}$, the space of $B$-multipliers between $X_{E_{2}}$ and $X_{E_{3}}$, to be the set of sequences $\left(\lambda_{j}\right)_{j} \in E_{1}^{\mathbb{N}}$ such that $\left(B\left(\lambda_{j}, \hat{x}(j)\right)\right)_{j} \in X_{E_{3}}$ for all $(\hat{x}(j))_{j} \in X_{E_{2}}$, and we define the Hadamard projective tensor product $X_{E_{1} \circledast_{B}} X_{E_{2}}$ as consisting of those elements in $E_{3}^{\mathbb{N}}$ that can be represented as $\sum_{n} \sum_{j} B\left(\hat{x}_{n}(j), \hat{y}_{n}(j)\right)$, where $\left(x_{n}\right)_{n} \in X_{E_{1}},\left(y_{n}\right)_{n} \in X_{E_{2}}$, and $\sum_{n}\left\|x_{n}\right\|_{X_{E_{1}}}\left\|y_{n}\right\|_{X_{E_{2}}}<\infty$.

We will analyze some properties of these two spaces, relate them, and compute the Hadamard tensor products and the spaces of vector-valued multipliers in several cases, getting applications in the particular case where $E=\mathcal{L}\left(E_{1}, E_{2}\right)$ and $B(T, x)=T(x)$.
\end{abstract}

\section{INTRODUCTION AND PRELIMINARIES}

One of the classic problems in Fourier analysis is the description of the space of coefficient multipliers between function spaces. Several papers have shown mathematicians' interest in determining this space in particular cases (see the recent monograph [18]; see also [20] for the historical situation for Hardy spaces and [16] and [17] for several techniques and results regarding mixed norm; we refer the

Copyright 2016 by the Tusi Mathematical Research Group.

Received Dec. 11, 2014; Accepted Apr. 14, 2015.

${ }^{*}$ Corresponding author.

2010 Mathematics Subject Classification. Primary 46B28; Secondary 46E40.

Keywords. vector-valued multipliers, Hadamard product, bilinear map. 


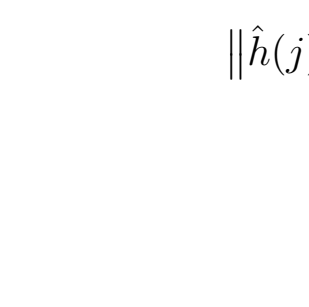

Banach J. Math. Anal. 10 (2016), no. 1, 71-95

http://dx.doi.org/10.1215/17358787-3319378

ISSN: $1735-8787$ (electronic)

http://projecteuclid.org/bjma

\title{
MULTIPLIERS AND HADAMARD PRODUCTS IN THE VECTOR-VALUED SETTING
}

\author{
ÓSCAR BLASCO and CARME ZARAGOZA-BERZOSA*
}

Communicated by R. Villena

\begin{abstract}
Let $E_{i}$ be Banach spaces, and let $X_{E_{i}}$ be Banach spaces continuously contained in the spaces of $E_{i}$-valued sequences $(\hat{x}(j))_{j} \in E_{i}^{\mathbb{N}}$, for $i=1,2,3$. Given a bounded bilinear map $B: E_{1} \times E_{2} \rightarrow E_{3}$, we define $\left(X_{E_{2}}, X_{E_{3}}\right)_{B}$, the space of $B$-multipliers between $X_{E_{2}}$ and $X_{E_{3}}$, to be the set of sequences $\left(\lambda_{j}\right)_{j} \in E_{1}^{\mathbb{N}}$ such that $\left(B\left(\lambda_{j}, \hat{x}(j)\right)\right)_{j} \in X_{E_{3}}$ for all $(\hat{x}(j))_{j} \in X_{E_{2}}$, and we define the Hadamard projective tensor product $X_{E_{1} \circledast_{B}} X_{E_{2}}$ as consisting of those elements in $E_{3}^{\mathbb{N}}$ that can be represented as $\sum_{n} \sum_{j} B\left(\hat{x}_{n}(j), \hat{y}_{n}(j)\right)$, where $\left(x_{n}\right)_{n} \in X_{E_{1}},\left(y_{n}\right)_{n} \in X_{E_{2}}$, and $\sum_{n}\left\|x_{n}\right\|_{X_{E_{1}}}\left\|y_{n}\right\|_{X_{E_{2}}}<\infty$.

We will analyze some properties of these two spaces, relate them, and compute the Hadamard tensor products and the spaces of vector-valued multipliers in several cases, getting applications in the particular case where $E=\mathcal{L}\left(E_{1}, E_{2}\right)$ and $B(T, x)=T(x)$.
\end{abstract}

\section{INTRODUCTION AND PRELIMINARIES}

One of the classic problems in Fourier analysis is the description of the space of coefficient multipliers between function spaces. Several papers have shown mathematicians' interest in determining this space in particular cases (see the recent monograph [18]; see also [20] for the historical situation for Hardy spaces and [16] and [17] for several techniques and results regarding mixed norm; we refer the

Copyright 2016 by the Tusi Mathematical Research Group.

Received Dec. 11, 2014; Accepted Apr. 14, 2015.

${ }^{*}$ Corresponding author.

2010 Mathematics Subject Classification. Primary 46B28; Secondary 46E40.

Keywords. vector-valued multipliers, Hadamard product, bilinear map. 


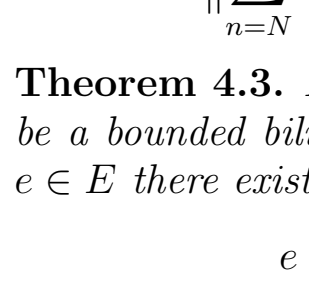

Banach J. Math. Anal. 10 (2016), no. 1, 71-95

http://dx.doi.org/10.1215/17358787-3319378

ISSN: $1735-8787$ (electronic)

http://projecteuclid.org/bjma

\title{
MULTIPLIERS AND HADAMARD PRODUCTS IN THE VECTOR-VALUED SETTING
}

\author{
ÓSCAR BLASCO and CARME ZARAGOZA-BERZOSA*
}

Communicated by R. Villena

\begin{abstract}
Let $E_{i}$ be Banach spaces, and let $X_{E_{i}}$ be Banach spaces continuously contained in the spaces of $E_{i}$-valued sequences $(\hat{x}(j))_{j} \in E_{i}^{\mathbb{N}}$, for $i=1,2,3$. Given a bounded bilinear map $B: E_{1} \times E_{2} \rightarrow E_{3}$, we define $\left(X_{E_{2}}, X_{E_{3}}\right)_{B}$, the space of $B$-multipliers between $X_{E_{2}}$ and $X_{E_{3}}$, to be the set of sequences $\left(\lambda_{j}\right)_{j} \in E_{1}^{\mathbb{N}}$ such that $\left(B\left(\lambda_{j}, \hat{x}(j)\right)\right)_{j} \in X_{E_{3}}$ for all $(\hat{x}(j))_{j} \in X_{E_{2}}$, and we define the Hadamard projective tensor product $X_{E_{1} \circledast_{B}} X_{E_{2}}$ as consisting of those elements in $E_{3}^{\mathbb{N}}$ that can be represented as $\sum_{n} \sum_{j} B\left(\hat{x}_{n}(j), \hat{y}_{n}(j)\right)$, where $\left(x_{n}\right)_{n} \in X_{E_{1}},\left(y_{n}\right)_{n} \in X_{E_{2}}$, and $\sum_{n}\left\|x_{n}\right\|_{X_{E_{1}}}\left\|y_{n}\right\|_{X_{E_{2}}}<\infty$.

We will analyze some properties of these two spaces, relate them, and compute the Hadamard tensor products and the spaces of vector-valued multipliers in several cases, getting applications in the particular case where $E=\mathcal{L}\left(E_{1}, E_{2}\right)$ and $B(T, x)=T(x)$.
\end{abstract}

\section{INTRODUCTION AND PRELIMINARIES}

One of the classic problems in Fourier analysis is the description of the space of coefficient multipliers between function spaces. Several papers have shown mathematicians' interest in determining this space in particular cases (see the recent monograph [18]; see also [20] for the historical situation for Hardy spaces and [16] and [17] for several techniques and results regarding mixed norm; we refer the

Copyright 2016 by the Tusi Mathematical Research Group.

Received Dec. 11, 2014; Accepted Apr. 14, 2015.

${ }^{*}$ Corresponding author.

2010 Mathematics Subject Classification. Primary 46B28; Secondary 46E40.

Keywords. vector-valued multipliers, Hadamard product, bilinear map. 


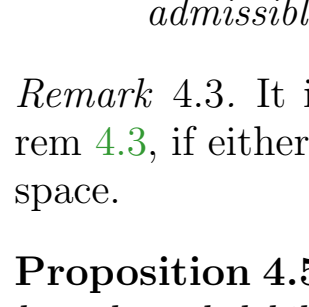

Banach J. Math. Anal. 10 (2016), no. 1, 71-95

http://dx.doi.org/10.1215/17358787-3319378

ISSN: $1735-8787$ (electronic)

http://projecteuclid.org/bjma

\title{
MULTIPLIERS AND HADAMARD PRODUCTS IN THE VECTOR-VALUED SETTING
}

\author{
ÓSCAR BLASCO and CARME ZARAGOZA-BERZOSA*
}

Communicated by R. Villena

\begin{abstract}
Let $E_{i}$ be Banach spaces, and let $X_{E_{i}}$ be Banach spaces continuously contained in the spaces of $E_{i}$-valued sequences $(\hat{x}(j))_{j} \in E_{i}^{\mathbb{N}}$, for $i=1,2,3$. Given a bounded bilinear map $B: E_{1} \times E_{2} \rightarrow E_{3}$, we define $\left(X_{E_{2}}, X_{E_{3}}\right)_{B}$, the space of $B$-multipliers between $X_{E_{2}}$ and $X_{E_{3}}$, to be the set of sequences $\left(\lambda_{j}\right)_{j} \in E_{1}^{\mathbb{N}}$ such that $\left(B\left(\lambda_{j}, \hat{x}(j)\right)\right)_{j} \in X_{E_{3}}$ for all $(\hat{x}(j))_{j} \in X_{E_{2}}$, and we define the Hadamard projective tensor product $X_{E_{1} \circledast_{B}} X_{E_{2}}$ as consisting of those elements in $E_{3}^{\mathbb{N}}$ that can be represented as $\sum_{n} \sum_{j} B\left(\hat{x}_{n}(j), \hat{y}_{n}(j)\right)$, where $\left(x_{n}\right)_{n} \in X_{E_{1}},\left(y_{n}\right)_{n} \in X_{E_{2}}$, and $\sum_{n}\left\|x_{n}\right\|_{X_{E_{1}}}\left\|y_{n}\right\|_{X_{E_{2}}}<\infty$.

We will analyze some properties of these two spaces, relate them, and compute the Hadamard tensor products and the spaces of vector-valued multipliers in several cases, getting applications in the particular case where $E=\mathcal{L}\left(E_{1}, E_{2}\right)$ and $B(T, x)=T(x)$.
\end{abstract}

\section{INTRODUCTION AND PRELIMINARIES}

One of the classic problems in Fourier analysis is the description of the space of coefficient multipliers between function spaces. Several papers have shown mathematicians' interest in determining this space in particular cases (see the recent monograph [18]; see also [20] for the historical situation for Hardy spaces and [16] and [17] for several techniques and results regarding mixed norm; we refer the

Copyright 2016 by the Tusi Mathematical Research Group.

Received Dec. 11, 2014; Accepted Apr. 14, 2015.

${ }^{*}$ Corresponding author.

2010 Mathematics Subject Classification. Primary 46B28; Secondary 46E40.

Keywords. vector-valued multipliers, Hadamard product, bilinear map. 


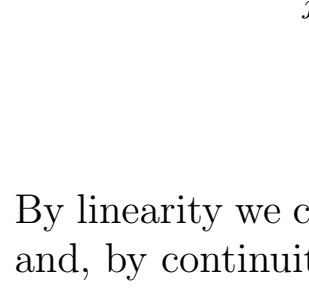

Banach J. Math. Anal. 10 (2016), no. 1, 71-95

http://dx.doi.org/10.1215/17358787-3319378

ISSN: $1735-8787$ (electronic)

http://projecteuclid.org/bjma

\title{
MULTIPLIERS AND HADAMARD PRODUCTS IN THE VECTOR-VALUED SETTING
}

\author{
ÓSCAR BLASCO and CARME ZARAGOZA-BERZOSA*
}

Communicated by R. Villena

\begin{abstract}
Let $E_{i}$ be Banach spaces, and let $X_{E_{i}}$ be Banach spaces continuously contained in the spaces of $E_{i}$-valued sequences $(\hat{x}(j))_{j} \in E_{i}^{\mathbb{N}}$, for $i=1,2,3$. Given a bounded bilinear map $B: E_{1} \times E_{2} \rightarrow E_{3}$, we define $\left(X_{E_{2}}, X_{E_{3}}\right)_{B}$, the space of $B$-multipliers between $X_{E_{2}}$ and $X_{E_{3}}$, to be the set of sequences $\left(\lambda_{j}\right)_{j} \in E_{1}^{\mathbb{N}}$ such that $\left(B\left(\lambda_{j}, \hat{x}(j)\right)\right)_{j} \in X_{E_{3}}$ for all $(\hat{x}(j))_{j} \in X_{E_{2}}$, and we define the Hadamard projective tensor product $X_{E_{1} \circledast_{B}} X_{E_{2}}$ as consisting of those elements in $E_{3}^{\mathbb{N}}$ that can be represented as $\sum_{n} \sum_{j} B\left(\hat{x}_{n}(j), \hat{y}_{n}(j)\right)$, where $\left(x_{n}\right)_{n} \in X_{E_{1}},\left(y_{n}\right)_{n} \in X_{E_{2}}$, and $\sum_{n}\left\|x_{n}\right\|_{X_{E_{1}}}\left\|y_{n}\right\|_{X_{E_{2}}}<\infty$.

We will analyze some properties of these two spaces, relate them, and compute the Hadamard tensor products and the spaces of vector-valued multipliers in several cases, getting applications in the particular case where $E=\mathcal{L}\left(E_{1}, E_{2}\right)$ and $B(T, x)=T(x)$.
\end{abstract}

\section{INTRODUCTION AND PRELIMINARIES}

One of the classic problems in Fourier analysis is the description of the space of coefficient multipliers between function spaces. Several papers have shown mathematicians' interest in determining this space in particular cases (see the recent monograph [18]; see also [20] for the historical situation for Hardy spaces and [16] and [17] for several techniques and results regarding mixed norm; we refer the

Copyright 2016 by the Tusi Mathematical Research Group.

Received Dec. 11, 2014; Accepted Apr. 14, 2015.

${ }^{*}$ Corresponding author.

2010 Mathematics Subject Classification. Primary 46B28; Secondary 46E40.

Keywords. vector-valued multipliers, Hadamard product, bilinear map. 


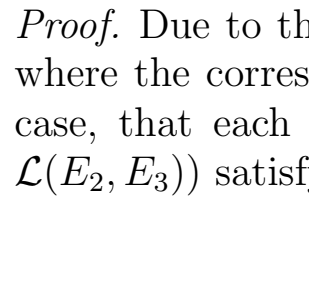

Banach J. Math. Anal. 10 (2016), no. 1, 71-95

http://dx.doi.org/10.1215/17358787-3319378

ISSN: $1735-8787$ (electronic)

http://projecteuclid.org/bjma

\title{
MULTIPLIERS AND HADAMARD PRODUCTS IN THE VECTOR-VALUED SETTING
}

\author{
ÓSCAR BLASCO and CARME ZARAGOZA-BERZOSA*
}

Communicated by R. Villena

\begin{abstract}
Let $E_{i}$ be Banach spaces, and let $X_{E_{i}}$ be Banach spaces continuously contained in the spaces of $E_{i}$-valued sequences $(\hat{x}(j))_{j} \in E_{i}^{\mathbb{N}}$, for $i=1,2,3$. Given a bounded bilinear map $B: E_{1} \times E_{2} \rightarrow E_{3}$, we define $\left(X_{E_{2}}, X_{E_{3}}\right)_{B}$, the space of $B$-multipliers between $X_{E_{2}}$ and $X_{E_{3}}$, to be the set of sequences $\left(\lambda_{j}\right)_{j} \in E_{1}^{\mathbb{N}}$ such that $\left(B\left(\lambda_{j}, \hat{x}(j)\right)\right)_{j} \in X_{E_{3}}$ for all $(\hat{x}(j))_{j} \in X_{E_{2}}$, and we define the Hadamard projective tensor product $X_{E_{1} \circledast_{B}} X_{E_{2}}$ as consisting of those elements in $E_{3}^{\mathbb{N}}$ that can be represented as $\sum_{n} \sum_{j} B\left(\hat{x}_{n}(j), \hat{y}_{n}(j)\right)$, where $\left(x_{n}\right)_{n} \in X_{E_{1}},\left(y_{n}\right)_{n} \in X_{E_{2}}$, and $\sum_{n}\left\|x_{n}\right\|_{X_{E_{1}}}\left\|y_{n}\right\|_{X_{E_{2}}}<\infty$.

We will analyze some properties of these two spaces, relate them, and compute the Hadamard tensor products and the spaces of vector-valued multipliers in several cases, getting applications in the particular case where $E=\mathcal{L}\left(E_{1}, E_{2}\right)$ and $B(T, x)=T(x)$.
\end{abstract}

\section{INTRODUCTION AND PRELIMINARIES}

One of the classic problems in Fourier analysis is the description of the space of coefficient multipliers between function spaces. Several papers have shown mathematicians' interest in determining this space in particular cases (see the recent monograph [18]; see also [20] for the historical situation for Hardy spaces and [16] and [17] for several techniques and results regarding mixed norm; we refer the

Copyright 2016 by the Tusi Mathematical Research Group.

Received Dec. 11, 2014; Accepted Apr. 14, 2015.

${ }^{*}$ Corresponding author.

2010 Mathematics Subject Classification. Primary 46B28; Secondary 46E40.

Keywords. vector-valued multipliers, Hadamard product, bilinear map. 


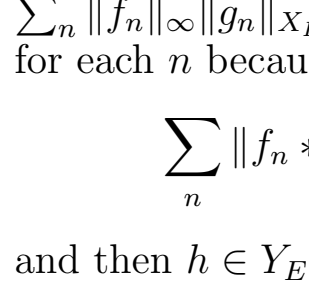

Banach J. Math. Anal. 10 (2016), no. 1, 71-95

http://dx.doi.org/10.1215/17358787-3319378

ISSN: $1735-8787$ (electronic)

http://projecteuclid.org/bjma

\title{
MULTIPLIERS AND HADAMARD PRODUCTS IN THE VECTOR-VALUED SETTING
}

\author{
ÓSCAR BLASCO and CARME ZARAGOZA-BERZOSA*
}

Communicated by R. Villena

\begin{abstract}
Let $E_{i}$ be Banach spaces, and let $X_{E_{i}}$ be Banach spaces continuously contained in the spaces of $E_{i}$-valued sequences $(\hat{x}(j))_{j} \in E_{i}^{\mathbb{N}}$, for $i=1,2,3$. Given a bounded bilinear map $B: E_{1} \times E_{2} \rightarrow E_{3}$, we define $\left(X_{E_{2}}, X_{E_{3}}\right)_{B}$, the space of $B$-multipliers between $X_{E_{2}}$ and $X_{E_{3}}$, to be the set of sequences $\left(\lambda_{j}\right)_{j} \in E_{1}^{\mathbb{N}}$ such that $\left(B\left(\lambda_{j}, \hat{x}(j)\right)\right)_{j} \in X_{E_{3}}$ for all $(\hat{x}(j))_{j} \in X_{E_{2}}$, and we define the Hadamard projective tensor product $X_{E_{1} \circledast_{B}} X_{E_{2}}$ as consisting of those elements in $E_{3}^{\mathbb{N}}$ that can be represented as $\sum_{n} \sum_{j} B\left(\hat{x}_{n}(j), \hat{y}_{n}(j)\right)$, where $\left(x_{n}\right)_{n} \in X_{E_{1}},\left(y_{n}\right)_{n} \in X_{E_{2}}$, and $\sum_{n}\left\|x_{n}\right\|_{X_{E_{1}}}\left\|y_{n}\right\|_{X_{E_{2}}}<\infty$.

We will analyze some properties of these two spaces, relate them, and compute the Hadamard tensor products and the spaces of vector-valued multipliers in several cases, getting applications in the particular case where $E=\mathcal{L}\left(E_{1}, E_{2}\right)$ and $B(T, x)=T(x)$.
\end{abstract}

\section{INTRODUCTION AND PRELIMINARIES}

One of the classic problems in Fourier analysis is the description of the space of coefficient multipliers between function spaces. Several papers have shown mathematicians' interest in determining this space in particular cases (see the recent monograph [18]; see also [20] for the historical situation for Hardy spaces and [16] and [17] for several techniques and results regarding mixed norm; we refer the

Copyright 2016 by the Tusi Mathematical Research Group.

Received Dec. 11, 2014; Accepted Apr. 14, 2015.

${ }^{*}$ Corresponding author.

2010 Mathematics Subject Classification. Primary 46B28; Secondary 46E40.

Keywords. vector-valued multipliers, Hadamard product, bilinear map. 


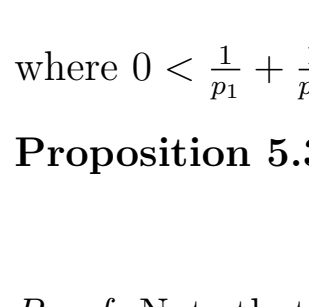

Banach J. Math. Anal. 10 (2016), no. 1, 71-95

http://dx.doi.org/10.1215/17358787-3319378

ISSN: $1735-8787$ (electronic)

http://projecteuclid.org/bjma

\title{
MULTIPLIERS AND HADAMARD PRODUCTS IN THE VECTOR-VALUED SETTING
}

\author{
ÓSCAR BLASCO and CARME ZARAGOZA-BERZOSA*
}

Communicated by R. Villena

\begin{abstract}
Let $E_{i}$ be Banach spaces, and let $X_{E_{i}}$ be Banach spaces continuously contained in the spaces of $E_{i}$-valued sequences $(\hat{x}(j))_{j} \in E_{i}^{\mathbb{N}}$, for $i=1,2,3$. Given a bounded bilinear map $B: E_{1} \times E_{2} \rightarrow E_{3}$, we define $\left(X_{E_{2}}, X_{E_{3}}\right)_{B}$, the space of $B$-multipliers between $X_{E_{2}}$ and $X_{E_{3}}$, to be the set of sequences $\left(\lambda_{j}\right)_{j} \in E_{1}^{\mathbb{N}}$ such that $\left(B\left(\lambda_{j}, \hat{x}(j)\right)\right)_{j} \in X_{E_{3}}$ for all $(\hat{x}(j))_{j} \in X_{E_{2}}$, and we define the Hadamard projective tensor product $X_{E_{1} \circledast_{B}} X_{E_{2}}$ as consisting of those elements in $E_{3}^{\mathbb{N}}$ that can be represented as $\sum_{n} \sum_{j} B\left(\hat{x}_{n}(j), \hat{y}_{n}(j)\right)$, where $\left(x_{n}\right)_{n} \in X_{E_{1}},\left(y_{n}\right)_{n} \in X_{E_{2}}$, and $\sum_{n}\left\|x_{n}\right\|_{X_{E_{1}}}\left\|y_{n}\right\|_{X_{E_{2}}}<\infty$.

We will analyze some properties of these two spaces, relate them, and compute the Hadamard tensor products and the spaces of vector-valued multipliers in several cases, getting applications in the particular case where $E=\mathcal{L}\left(E_{1}, E_{2}\right)$ and $B(T, x)=T(x)$.
\end{abstract}

\section{INTRODUCTION AND PRELIMINARIES}

One of the classic problems in Fourier analysis is the description of the space of coefficient multipliers between function spaces. Several papers have shown mathematicians' interest in determining this space in particular cases (see the recent monograph [18]; see also [20] for the historical situation for Hardy spaces and [16] and [17] for several techniques and results regarding mixed norm; we refer the

Copyright 2016 by the Tusi Mathematical Research Group.

Received Dec. 11, 2014; Accepted Apr. 14, 2015.

${ }^{*}$ Corresponding author.

2010 Mathematics Subject Classification. Primary 46B28; Secondary 46E40.

Keywords. vector-valued multipliers, Hadamard product, bilinear map. 


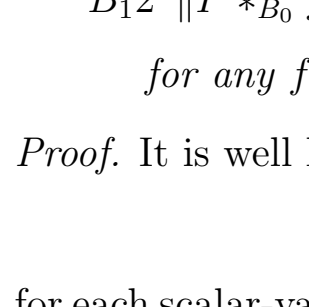

Banach J. Math. Anal. 10 (2016), no. 1, 71-95

http://dx.doi.org/10.1215/17358787-3319378

ISSN: $1735-8787$ (electronic)

http://projecteuclid.org/bjma

\title{
MULTIPLIERS AND HADAMARD PRODUCTS IN THE VECTOR-VALUED SETTING
}

\author{
ÓSCAR BLASCO and CARME ZARAGOZA-BERZOSA*
}

Communicated by R. Villena

\begin{abstract}
Let $E_{i}$ be Banach spaces, and let $X_{E_{i}}$ be Banach spaces continuously contained in the spaces of $E_{i}$-valued sequences $(\hat{x}(j))_{j} \in E_{i}^{\mathbb{N}}$, for $i=1,2,3$. Given a bounded bilinear map $B: E_{1} \times E_{2} \rightarrow E_{3}$, we define $\left(X_{E_{2}}, X_{E_{3}}\right)_{B}$, the space of $B$-multipliers between $X_{E_{2}}$ and $X_{E_{3}}$, to be the set of sequences $\left(\lambda_{j}\right)_{j} \in E_{1}^{\mathbb{N}}$ such that $\left(B\left(\lambda_{j}, \hat{x}(j)\right)\right)_{j} \in X_{E_{3}}$ for all $(\hat{x}(j))_{j} \in X_{E_{2}}$, and we define the Hadamard projective tensor product $X_{E_{1} \circledast_{B}} X_{E_{2}}$ as consisting of those elements in $E_{3}^{\mathbb{N}}$ that can be represented as $\sum_{n} \sum_{j} B\left(\hat{x}_{n}(j), \hat{y}_{n}(j)\right)$, where $\left(x_{n}\right)_{n} \in X_{E_{1}},\left(y_{n}\right)_{n} \in X_{E_{2}}$, and $\sum_{n}\left\|x_{n}\right\|_{X_{E_{1}}}\left\|y_{n}\right\|_{X_{E_{2}}}<\infty$.

We will analyze some properties of these two spaces, relate them, and compute the Hadamard tensor products and the spaces of vector-valued multipliers in several cases, getting applications in the particular case where $E=\mathcal{L}\left(E_{1}, E_{2}\right)$ and $B(T, x)=T(x)$.
\end{abstract}

\section{INTRODUCTION AND PRELIMINARIES}

One of the classic problems in Fourier analysis is the description of the space of coefficient multipliers between function spaces. Several papers have shown mathematicians' interest in determining this space in particular cases (see the recent monograph [18]; see also [20] for the historical situation for Hardy spaces and [16] and [17] for several techniques and results regarding mixed norm; we refer the

Copyright 2016 by the Tusi Mathematical Research Group.

Received Dec. 11, 2014; Accepted Apr. 14, 2015.

${ }^{*}$ Corresponding author.

2010 Mathematics Subject Classification. Primary 46B28; Secondary 46E40.

Keywords. vector-valued multipliers, Hadamard product, bilinear map. 


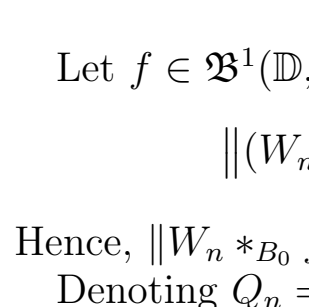

Banach J. Math. Anal. 10 (2016), no. 1, 71-95

http://dx.doi.org/10.1215/17358787-3319378

ISSN: $1735-8787$ (electronic)

http://projecteuclid.org/bjma

\title{
MULTIPLIERS AND HADAMARD PRODUCTS IN THE VECTOR-VALUED SETTING
}

\author{
ÓSCAR BLASCO and CARME ZARAGOZA-BERZOSA*
}

Communicated by R. Villena

\begin{abstract}
Let $E_{i}$ be Banach spaces, and let $X_{E_{i}}$ be Banach spaces continuously contained in the spaces of $E_{i}$-valued sequences $(\hat{x}(j))_{j} \in E_{i}^{\mathbb{N}}$, for $i=1,2,3$. Given a bounded bilinear map $B: E_{1} \times E_{2} \rightarrow E_{3}$, we define $\left(X_{E_{2}}, X_{E_{3}}\right)_{B}$, the space of $B$-multipliers between $X_{E_{2}}$ and $X_{E_{3}}$, to be the set of sequences $\left(\lambda_{j}\right)_{j} \in E_{1}^{\mathbb{N}}$ such that $\left(B\left(\lambda_{j}, \hat{x}(j)\right)\right)_{j} \in X_{E_{3}}$ for all $(\hat{x}(j))_{j} \in X_{E_{2}}$, and we define the Hadamard projective tensor product $X_{E_{1} \circledast_{B}} X_{E_{2}}$ as consisting of those elements in $E_{3}^{\mathbb{N}}$ that can be represented as $\sum_{n} \sum_{j} B\left(\hat{x}_{n}(j), \hat{y}_{n}(j)\right)$, where $\left(x_{n}\right)_{n} \in X_{E_{1}},\left(y_{n}\right)_{n} \in X_{E_{2}}$, and $\sum_{n}\left\|x_{n}\right\|_{X_{E_{1}}}\left\|y_{n}\right\|_{X_{E_{2}}}<\infty$.

We will analyze some properties of these two spaces, relate them, and compute the Hadamard tensor products and the spaces of vector-valued multipliers in several cases, getting applications in the particular case where $E=\mathcal{L}\left(E_{1}, E_{2}\right)$ and $B(T, x)=T(x)$.
\end{abstract}

\section{INTRODUCTION AND PRELIMINARIES}

One of the classic problems in Fourier analysis is the description of the space of coefficient multipliers between function spaces. Several papers have shown mathematicians' interest in determining this space in particular cases (see the recent monograph [18]; see also [20] for the historical situation for Hardy spaces and [16] and [17] for several techniques and results regarding mixed norm; we refer the

Copyright 2016 by the Tusi Mathematical Research Group.

Received Dec. 11, 2014; Accepted Apr. 14, 2015.

${ }^{*}$ Corresponding author.

2010 Mathematics Subject Classification. Primary 46B28; Secondary 46E40.

Keywords. vector-valued multipliers, Hadamard product, bilinear map. 


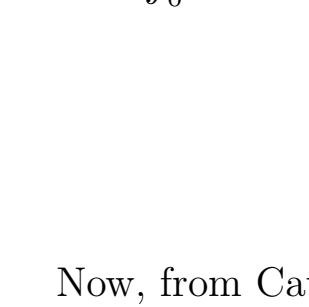

Banach J. Math. Anal. 10 (2016), no. 1, 71-95

http://dx.doi.org/10.1215/17358787-3319378

ISSN: $1735-8787$ (electronic)

http://projecteuclid.org/bjma

\title{
MULTIPLIERS AND HADAMARD PRODUCTS IN THE VECTOR-VALUED SETTING
}

\author{
ÓSCAR BLASCO and CARME ZARAGOZA-BERZOSA*
}

Communicated by R. Villena

\begin{abstract}
Let $E_{i}$ be Banach spaces, and let $X_{E_{i}}$ be Banach spaces continuously contained in the spaces of $E_{i}$-valued sequences $(\hat{x}(j))_{j} \in E_{i}^{\mathbb{N}}$, for $i=1,2,3$. Given a bounded bilinear map $B: E_{1} \times E_{2} \rightarrow E_{3}$, we define $\left(X_{E_{2}}, X_{E_{3}}\right)_{B}$, the space of $B$-multipliers between $X_{E_{2}}$ and $X_{E_{3}}$, to be the set of sequences $\left(\lambda_{j}\right)_{j} \in E_{1}^{\mathbb{N}}$ such that $\left(B\left(\lambda_{j}, \hat{x}(j)\right)\right)_{j} \in X_{E_{3}}$ for all $(\hat{x}(j))_{j} \in X_{E_{2}}$, and we define the Hadamard projective tensor product $X_{E_{1} \circledast_{B}} X_{E_{2}}$ as consisting of those elements in $E_{3}^{\mathbb{N}}$ that can be represented as $\sum_{n} \sum_{j} B\left(\hat{x}_{n}(j), \hat{y}_{n}(j)\right)$, where $\left(x_{n}\right)_{n} \in X_{E_{1}},\left(y_{n}\right)_{n} \in X_{E_{2}}$, and $\sum_{n}\left\|x_{n}\right\|_{X_{E_{1}}}\left\|y_{n}\right\|_{X_{E_{2}}}<\infty$.

We will analyze some properties of these two spaces, relate them, and compute the Hadamard tensor products and the spaces of vector-valued multipliers in several cases, getting applications in the particular case where $E=\mathcal{L}\left(E_{1}, E_{2}\right)$ and $B(T, x)=T(x)$.
\end{abstract}

\section{INTRODUCTION AND PRELIMINARIES}

One of the classic problems in Fourier analysis is the description of the space of coefficient multipliers between function spaces. Several papers have shown mathematicians' interest in determining this space in particular cases (see the recent monograph [18]; see also [20] for the historical situation for Hardy spaces and [16] and [17] for several techniques and results regarding mixed norm; we refer the

Copyright 2016 by the Tusi Mathematical Research Group.

Received Dec. 11, 2014; Accepted Apr. 14, 2015.

${ }^{*}$ Corresponding author.

2010 Mathematics Subject Classification. Primary 46B28; Secondary 46E40.

Keywords. vector-valued multipliers, Hadamard product, bilinear map. 


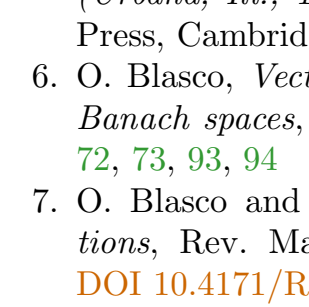

Banach J. Math. Anal. 10 (2016), no. 1, 71-95

http://dx.doi.org/10.1215/17358787-3319378

ISSN: $1735-8787$ (electronic)

http://projecteuclid.org/bjma

\title{
MULTIPLIERS AND HADAMARD PRODUCTS IN THE VECTOR-VALUED SETTING
}

\author{
ÓSCAR BLASCO and CARME ZARAGOZA-BERZOSA*
}

Communicated by R. Villena

\begin{abstract}
Let $E_{i}$ be Banach spaces, and let $X_{E_{i}}$ be Banach spaces continuously contained in the spaces of $E_{i}$-valued sequences $(\hat{x}(j))_{j} \in E_{i}^{\mathbb{N}}$, for $i=1,2,3$. Given a bounded bilinear map $B: E_{1} \times E_{2} \rightarrow E_{3}$, we define $\left(X_{E_{2}}, X_{E_{3}}\right)_{B}$, the space of $B$-multipliers between $X_{E_{2}}$ and $X_{E_{3}}$, to be the set of sequences $\left(\lambda_{j}\right)_{j} \in E_{1}^{\mathbb{N}}$ such that $\left(B\left(\lambda_{j}, \hat{x}(j)\right)\right)_{j} \in X_{E_{3}}$ for all $(\hat{x}(j))_{j} \in X_{E_{2}}$, and we define the Hadamard projective tensor product $X_{E_{1} \circledast_{B}} X_{E_{2}}$ as consisting of those elements in $E_{3}^{\mathbb{N}}$ that can be represented as $\sum_{n} \sum_{j} B\left(\hat{x}_{n}(j), \hat{y}_{n}(j)\right)$, where $\left(x_{n}\right)_{n} \in X_{E_{1}},\left(y_{n}\right)_{n} \in X_{E_{2}}$, and $\sum_{n}\left\|x_{n}\right\|_{X_{E_{1}}}\left\|y_{n}\right\|_{X_{E_{2}}}<\infty$.

We will analyze some properties of these two spaces, relate them, and compute the Hadamard tensor products and the spaces of vector-valued multipliers in several cases, getting applications in the particular case where $E=\mathcal{L}\left(E_{1}, E_{2}\right)$ and $B(T, x)=T(x)$.
\end{abstract}

\section{INTRODUCTION AND PRELIMINARIES}

One of the classic problems in Fourier analysis is the description of the space of coefficient multipliers between function spaces. Several papers have shown mathematicians' interest in determining this space in particular cases (see the recent monograph [18]; see also [20] for the historical situation for Hardy spaces and [16] and [17] for several techniques and results regarding mixed norm; we refer the

Copyright 2016 by the Tusi Mathematical Research Group.

Received Dec. 11, 2014; Accepted Apr. 14, 2015.

${ }^{*}$ Corresponding author.

2010 Mathematics Subject Classification. Primary 46B28; Secondary 46E40.

Keywords. vector-valued multipliers, Hadamard product, bilinear map. 